\title{
Mechanisms and Implications of Age-Associated Impaired Innate Interferon Secretion by Dendritic Cells: A Mini-Review
}

\author{
Anshu Agrawal \\ Division of Basic and Clinical Immunology, Department of Medicine, University of California, Irvine, Calif., USA
}

\section{Key Words}

Dendritic cells · Aging · Type I interferon · Type III interferon

\begin{abstract}
Initial secretion of interferons by innate immune cells such as dendritic cells is crucial for protection against infections as well as for alerting and activating the downstream immune responses. The secretion of innate interferons, both type I and type III, by dendritic cells is severely impaired in aged subjects. This review focuses on the mechanisms responsible for the reduced interferon secretion by dendritic cells and the role this plays in the increased susceptibility of the elderly to infections, particularly of the respiratory mucosa.

Copyright $\odot 2013$ S. Karger AG, Basel
\end{abstract}

\section{Introduction}

The immune system undergoes significant changes with advancing age. Increased susceptibility to infections particularly to viral infections such as influenza, respiratory syncytial virus, West Nile virus and herpes zoster virus is the cause of significant morbidity and mortality in the elderly [1]. Production of innate interferons (IFNs) constitutes the most rapid and dominant innate immune response to counter viral infections.

\section{KARGER}

(c) 2013 S. Karger AG, Basel

0304-324X/13/0595-0421\$38.00/0

E-Mail karger@karger.com

www.karger.com/ger
IFNs are small single-chain glycoproteins that can be divided into three major classes, type I IFN (IFN- $\alpha, \beta, \omega)$, type II IFN (IFN- $\gamma$ ) and type III IFN (IFN- $\lambda$, IL28/29). Types I and III are produced primarily by innate immune cells such as dendritic cells (DCs), macrophages and epithelial cells and aid in preventing viral replication and activating downstream adaptive immune responses. The IFNs secreted early in response to viral infections affect the functions of other innate and adaptive immune cells by enhancing their activation and anti-microbial activity, and therefore are critical for clearing infections [2]. Ageassociated impaired IFN secretion thus may play a critical role in the increased susceptibility of the elderly to viral infections. This review focuses primarily on innate IFNs, types I and III, secreted by DCs.

\section{Aging, Plasmacytoid Dendritic Cells and Type I Interferon Secretion}

Amongst the innate immune cells, plasmacytoid dendritic cells (PDCs) are the most rapid and abundant IFN producers [3]. This is because of constitutive expression of IFN-regulatory factor-7 (IRF7), a transcription factor responsible for IFN production [4]. Because of the readily available machinery, PDCs can also produce up to 100fold more IFNs compared to other cells. In addition, PDCs express the unique intracellular Toll-like receptors (TLRs), 
TLR7 and TLR9 which are important in sensing viruses. TLR7 can sense single-stranded RNA (ssRNA) viruses such as influenza while TLR9 can sense unmethylated DNA sequences from pathogens. Signaling through these TLRs in PDCs leads to prompt secretion of IFNs which is crucial for activation of other innate immune cells and priming of downstream adaptive immune responses.

As PDCs play a crucial role in fighting viral infections, numerous studies have compared the functions of PDCs between the aged and young donors. The results are variable particularly with regards to PDC numbers in the elderly. Studies by Shodell and Siegal [5], Perez et al. [6], Jing et al. [7] and Canaday et al. [8] report a decline in PDC numbers in healthy elderly individuals. Jing et al. [7] found the numbers to be further compromised in the frail elderly population. In contrast, our own studies $[9,10]$ along with those of Della Bella et al. [11] did not observe any significant difference in PDC percentage in blood between healthy aged and young subjects. The may be a consequence of differences in the aged population. PDC numbers are also stable in mice and do not seem to change substantially with advancing age.

In contrast, the response of PDCs to viruses, particularly IFN production, is significantly impaired with age. Almost all studies report a significant decline in type I IFN production in response to various infections. Early studies by Shodell and Siegal [5] observed impaired type I IFN secretion from PBMCs from aged subjects compared to young after activation with ultraviolet-inactivated herpes simplex virus. They attributed it to reduced PDC numbers. Jing et al. [7] also observed reduced IFN- $\alpha$ secretion from purified aged PDCs after activation with influenza virus and TLR9 ligand, ODN2006. They also attributed the impaired IFN secretion to declining PDC numbers. In addition, the authors reported reduced expression of TLR7 and TLR9 in PDC which would further account for the reduced production of IFN. Studies by Canaday et al. [8] also documented significant decline in IFN production by PDCs from aged subjects in response to multiple strains of influenza virus. They also attributed it to reduced PDC numbers in aged subjects as no defects in TLR expression, uptake of influenza virus and viability of PDCs was observed in these studies. However, in contrast to Jing et al. [7], Canaday et al. [8] did not observe a reduction in IFN secretion from aged PDCs in response to ODN and the TLR7 ligand Gardiquimod. It could be because of the difference in methodology between the two studies. Canaday et al. [8] determined IFN secretion in whole PBMCs and observed that depletion of PDCs resulted in abrogation of IFN secretion. Impaired type I IFN production in re- sponse to TLR7/8 ligand R848, Gardiquimod and ODN was also observed by Panda et al. [12] in a large cohort of aged and young subjects. PDC numbers and TLR9 expression were comparable to young in their cohort but PDCTLR7 expression was decreased. This suggests that reduced IFN secretion in aged is a consequence of both transcriptional and post transcriptional mechanism such as age-associated modifications in signaling mechanism responsible for IFN production. This was confirmed by our own observations [10]. We reported impaired type I IFN secretion from purified aged PDCs after stimulation with ODN and influenza virus. Secretion of all other pro-inflammatory cytokines was comparable to young indicating that the initial interaction of the ligand with the receptor was not impaired. This conclusion was further supported by comparable PDC numbers and TLR7 and TLR9 expression in aged and young PDCs. This suggested that the defect lay in the signaling pathway involved in the production of IFN- $\alpha$ such as mTOR and IRF7. Both these pathways play a critical role in production of IFN by PDCs. The interaction of TLR7/9 with MyD88 in PDCs elicits the formation of multifactor signaling transduction complex that contains the kinases IRAK1 and IRAK4, the ubiquitin ligase TRAF6, and IRF7. IRF7 is phosphorylated by IRAK1-IRAK4 and then translocates to the nucleus, where it initiates the transcription of genes encoding type I IFNs. A recent study [13] demonstrated that activation of TLR7/9 on PDCs phosphorylates mTOR which leads to activation of p70S6K. This enhances the interaction of TLR with MyD88 and consequently promotes the phosphorylation of IRF7. Our investigations into the signaling mechanisms indicated that phosphorylation of IRF-7 was significantly impaired in aged PDCs. Defective IRF7 activation in PDCs has also been reported in aged mouse PDCs [14]. There is no report regarding the status of mTOR signaling in aged PDCs though it would not be surprising to find that this pathway is also affected in aged PDCs as mTOR signaling pathway is one of the pathways which is significantly affected by advancing age. It would be interesting to determine the status of IFN secretion by PDCs in aged mice treated with rapamycin, an inhibitor of mTOR signaling pathway. A recent study [15] reports similar deficiency in IFN production from aged PDCs in response to West Nile virus.

Almost all studies comparing the difference in PDC IFN secretion are cross-sectional; however, a very recent report by Uno et al. [16] utilizes a longitudinal cohort of Japanese healthy subjects where the IFN- $\alpha$ secretion in response to Sendai virus was determined twice a year in total PBMCs for 24 years. They observed that though the 
IFN-a secretion declined around age 55 , it reverted back to normal levels around age 65 . In subjects with other comorbidities such as diabetes, cancer the decline in IFN was irreversible. They therefore concluded that decline in the IFN production is more a function of the onset of disease rather than aging.

Though all aging studies concur on the impaired production of type I IFN by PDCs, the mechanisms responsible seem to be variable. These discrepancies could be due to differences in sample population as well as size of sample. In general, studies with healthy aged populations such as those living independently and free of comorbidities such as diabetes, etc. report fewer changes. Another reason could be differences in sample processing. PDCs are relatively rare cells and sufficient numbers of cells have to be acquired during flow cytometry to achieve statistically reliable data. Analysis using percentage of PDC versus absolute numbers may also result in differences. The problem could also be a consequence of various TLR antibody clones used as the specificity may vary. Thus, it seems that certain standard guidelines need to be established regarding sample processing and acquisition to allow comparison between different aging studies.

\section{Myeloid Dendritic Cell Type I Interferon Secretion}

In addition to PDCs, the myeloid dendritic cells (MDCs) subset is also capable of producing IFNs in response to viral infection. Though the magnitude of IFN secreted is significantly lower than PDC nevertheless, unlike PDCs, MDCs retain the ability to express IFNs for a long period of time after infection [2]. Viruses including influenza virus induce type I IFN secretion via multiple receptors in MDCs. Both TLR3 and TLR8 are known to be activated by influenza. TLR3 induces type I IFN via Toll-like IL-1 receptor-related adaptor protein inducing IFN (TRIF), which activates both IRF3 and NF- $\kappa$ B. TLR8 on the other hand signals via MyD88 adaptor protein and induces IFN secretion via IRF3. Besides TLRs, influenza also activates the cytosolic retinoic acid-inducible gene-I (RIG-I) encoded helicases to induce IFN via the IPS-1/MAV5 signaling pathway. An additional cytoplasmic RNA sensor, the RNA-activated protein kinase receptor, also binds dsRNA as well as structured ssRNAs that then mediate autophosphorylation and activation of the protein kinase receptor. Our previous studies had demonstrated that IRF3 phosphorylation and NF- $\kappa \mathrm{B}$ activation in response to self DNA is enhanced in aged MDCs [17]. Cytosolic sensors for self DNA are different from that utilized by viruses. It may be that IRF3 phosphorylation downstream of TLR3/8 is impaired with age. Studies by Panda et al. [12] have reported decreased expression and response of both these receptors in MDCs from aged. They observed decreased secretion of TNF- $\alpha$, IL-12p40 and IL-6 in response to TLR3 and TLR8 ligand. IFN secretion by MDCs was not determined in this study.

We investigated the capacity of aged MDCs to produce type I IFN in response to influenza virus [18]. This is because MDCs are comparatively more abundant than PDCs and IFN production by them may compensate for the reduced IFN by PDCs. Similar to PDCs, MDCs from aged individuals were also impaired in their capacity to produce type I IFN while the ability to produce other inflammatory cytokines was not affected. A similar reduction in the capacity of MDCs from older donors to produce IFN- $\alpha$ in response to West Nile virus has also been reported [15]. This is in contrast to self DNA where we observed an increased secretion of type I IFN by aged MDCs. The production of IFN secretion to self and foreign antigens seems to be differentially regulated in the aged. It will be interesting to investigate whether enhancement of one leads to deterioration of the other.

Recently, there has been an increased appreciation for the role of chromatin remodeling in gene regulation. Aging is associated with dramatic changes in chromatin structure; therefore, we investigated whether the impaired IFN secretion by aged DCs could be explained by age-associated changes in chromatin remodeling [18]. The association of IFN promoters to activator and repressor histones in MDCs was investigated using chromatin immunoprecipitation (ChIP). Increased association of the IFN promoter to repressor histone, $\mathrm{H} 3 \mathrm{~K} 9$, was observed in unactivated aged MDCs compared to unactivated young DCs. This led to the decreased association of the IFN promoters to activator histone, $\mathrm{H} 3 \mathrm{~K} 4$, after stimulation with influenza resulting in decreased IFN production. In contrast to IFN promoters, the binding of TNF promoter to both $\mathrm{H} 3 \mathrm{~K} 4$ and $\mathrm{H} 3 \mathrm{~K} 9$ histones was comparable in aged and young MDCs both before and after activation with influenza resulting in comparable levels of TNF production. In the case of West Nile virus, the authors observed a decreased expression of IRF1, suggesting a defective positive-feedback regulation of type I IFN expression [15].

Collectively, these findings suggest that the production of IFNs by DCs is significantly affected with age. Mechanism of impairment seem to be at all levels ranging from reduced DC numbers to reduced expression of receptors to impaired downstream signaling and also at the level of transcriptional regulation. 
Fig. 1. Effect of IFN on immune and other cells. Viruses such as influenza activate PDCs and MDCs to induce secretion of type I and type III IFN which activate and prime antiviral responses in various cells. In aged subjects, reduced secretion of types I and III IFN (red arrow) results in generation of inefficient innate and adaptive immune responses that are ineffective in fighting infections.

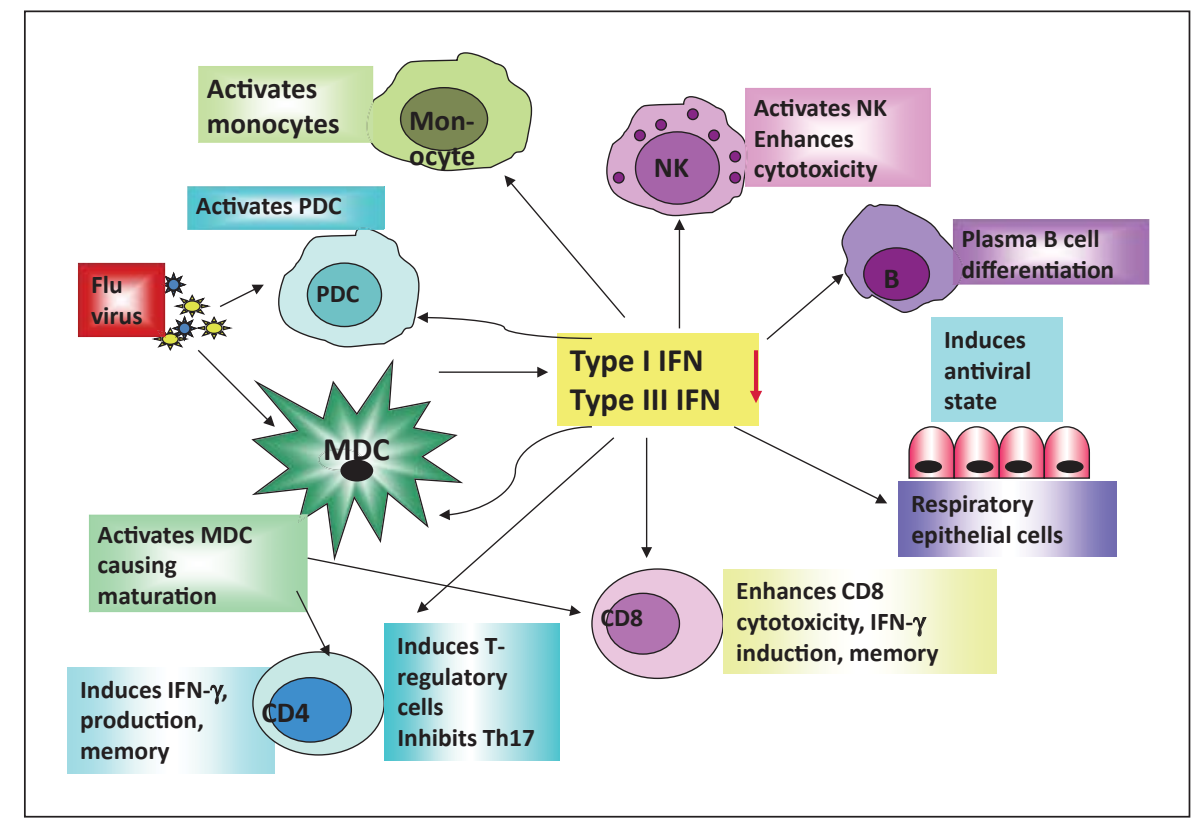

\section{Implications Type I Interferon and Immune Response}

Type I IFNs orchestrate numerous biological and cellular processes and are essential elements during host antiviral defense (fig. 1) [2, 19]. Humans with impaired type I IFN production and signaling display increased susceptibility to viral infections. Mice deficient in type I IFN are also unable to control a wide range of viral infections. Triggering of IFN production by viruses leads to the transcription of hundreds of IFN-stimulated genes. The products of these IFN-stimulated genes exert numerous antiviral effector functions, many of which are still not fully understood. IFNs modulate distinct aspects of both innate and adaptive immunity. In addition to directly interfering with viral replication, type I IFN act on antigenpresenting cells such as DCs and macrophages to amplify antigen presentation to specific $\mathrm{T}$ cells through the upregulation of MHC and costimulatory molecules. Furthermore, IFNs also promote DC generation from monocytes and stimulate DC maturation.

IFNs exert multiple direct and indirect effects on the adaptive immune system that impact almost every cell type and function [19]. Type I IFNs enhance the cytotoxicity of NK cells which play an important role in the early phase of immune response against viral, bacterial, and other microbial pathogens. NK cells constitute the aged population's major antiviral defense mechanism. It has been reported that low NK cytotoxic activity in the aged correlates with a history of severe infections or death due to infection. Decreased NK cell cytotoxic activity also is related to aged subjects' susceptibility to common infectious disease. Reduced IFN secretion by DCs may therefore be responsible for the poor priming of NK cells in the elderly.

Similar to NK cells, IFNs also enhance the cytotoxic activity of CD8 T cells. Several studies have demonstrated that type I IFNs play an essential role in effector CD8 Tcell differentiation and function. Murali-Krishna and colleagues [20] observed that CD8 T cells lacking the type I IFN receptor were profoundly impaired in their ability to expand and differentiate into effector CTLs, demonstrating that type I IFNs provide a nonredundant costimulatory signal in vivo. In addition, type I IFN enhances IFN- $\gamma$ secretion from both CD8 and CD4 T cells. Reduced type I IFN secretion in aging may therefore be a key culprit in the reduced response of the aged to viral infections. The antigen-specific cytotoxic T lymphocyte (CTL) response against pathogens is markedly reduced in an aged immune system. Studies from various groups have demonstrated that influenza virus-specific CTL activity was significantly diminished among elderly subjects when compared with young subjects. The aged population's CTL response against influenza vaccination is also reduced and short-lived. Therefore, diminished CTL activity in aged subjects may be responsible for poor protection against influenza infection, leading to prolonged and more severe infection. DCs constitute the first line of defense against bacterial and viral infections and play in critical role in 
generation of memory $\mathrm{T}$ cell responses. The initial weak priming of T cells by DCs from aged populations may lead to generation of weak or ineffective memory response.

In addition to CD8 T cells, type I IFNs also impact CD4 $\mathrm{T}$ cell functions. Type I IFN produced in response to viruses is reported to suppress $\mathrm{CD} 4 \mathrm{~T}$ cell responses via induction of IL-10 producing $\mathrm{T}$ regulatory cells. Indoleamine 2,3-dioxygenase (IDO) is an important enzyme in the tryptophan catabolism pathway and plays a key role in triggering T-regulatory cells [21]. Several studies document the reciprocal relationship between IDO expression and type I IFN production [22-24]. IDO increases the accumulation of kynurenine pathway metabolites, which suppress type I IFNs production and enhance viral replication [25]. Increased IDO activity in plasma has been observed in aged subjects as compared to young individuals [26] and has been linked to chronic inflammation, neuropsychiatric disorders and metabolic syndrome. Increased IDO expression may suppress $\mathrm{T}$ cell responsiveness and lead to age-associated immunodeficiency [27].

Besides T cells, type I IFN can also enhance antibody response to soluble antigens and promote immunoglobulin class switch and immunological memory as well as induce the differentiation of B cells in plasmablasts and subsequently into plasma cells. Aging is associated with reduced antibody response to all vaccines, including those for influenza, encephalitis, pneumococcal pneumonia, and tetanus. The cause of this decline in antibody responses remains an open question. The reduced IFN- $\alpha$ secretion of DCs in response to pathogens may be one of the causes of poor antibody responses. Efficient induction of cytotoxic $\mathrm{T}$ cell responses as well as B cell antibody responses is essential for providing effective immunity against viruses. Reduced IFN secretion by aged DCs may therefore lead to a reduction in adaptive immune $\mathrm{T}$ and $\mathrm{B}$ cell responses and increased susceptibility to infections.

\section{Type III IFNs}

In addition to type I IFNs, PDCs and MDCs also secrete type III IFNs. Type III IFNs are IFN- $\lambda 1$, IFN- $\lambda 2$ and IFN- $\lambda 3$, also known as IL-29, IL-28A, and IL-28B, respectively. They signal through an IFN- $\lambda$ receptor complex composed of a unique IFN- $\lambda \mathrm{R} 1$ chain and a shared IL10R2 chain that is also the second subunit of the IL-10, IL-22, and IL-26 receptor complexes [28]. Type III IFN, have been reported to possess antiviral activities and share common features with the type I IFNs [29]. Studies of the molecular events leading to IFN $\lambda$ production sug-

Innate Interferons and Aging gest a common mechanism with type I IFNs. Binding of IFNs to their corresponding cellular receptor complexes also induces similar signaling events activating a very similar set of antiviral genes. Consequently, type III and type I IFNs are both able to induce an antiviral state in cells. However, whereas type I IFN receptors are expressed in most cell types, IFN- $\lambda \mathrm{R} 1$ demonstrates a more restricted pattern of expression, limiting the response of type III IFNs to primarily epithelium-like tissues. In addition to differential action, there are also indications that IFN- $\lambda$ signaling may be resistant to feedback mechanisms targeting IFN- $\alpha$, allowing it to be a more effective antiviral due to enhanced production and prolonged action in the mucosal compartments that are exposed to the outside. Therefore, although both type I and type III IFNs share similar expression pattern and biological activities, they may play distinct roles in the establishment of multifaceted antiviral response. Experiments in vivo demonstrate that type III IFNs are important mediators of antiviral response in mucosal/epithelial tissues. It has been reported that type III IFN contributes greatly in defense against pathogens that infects respiratory tract such as influenza A virus, influenza B virus, and respiratory syncytial virus. Furthermore, IFN- $\lambda$ production was inversely correlated with the clinical severity of allergic asthma and airway inflammation. Koltsida et al. [30] reported that overexpression of recombinant or adenovirally expressed IL-28A in mice suppressed allergic airway disease. Their investigations demonstrated that type III IFN suppressed Th2 and Th17 responses and induced IFN- $\gamma$.

As all of these infections are highly prevalent in the elderly, we investigated the production of type III IFN by PDCs and MDCs as these cells are the primary producers of type III IFN. Similar to type I IFN, both PDC and MDC from aged subjects were severely impaired in their capacity to produce type III IFN in response to influenza virus [10, 17]. The magnitude of impairment was higher than that observed for type I IFN, suggesting that type III IFN deficiency may have a critical role to play in the increased susceptibility of aged to respiratory infections. Since mechanisms of induction of both type I and III IFNs are analogous, reduced IRF7 phosphorylation may be responsible for the decline in type III IFN production by aged PDCs. Akin to type I IFN, the promoter for type III IFN also displayed increased binding to repressor histone, H3K9me3 in aged MDCs which led to decreased association of the promoter to activator histone, $\mathrm{H} 3 \mathrm{~K} 4 \mathrm{me} 3$ after activation with influenza resulting in impaired type III IFN secretion.

In summary, these data suggest that the elderly display a significant deficiency in both type I IFN and type III IFN 
production. Treatment strategies restoring IFNs in the elderly may be effective in reducing the burden of respiratory diseases in the elderly. In this regard, type III IFN may be more effective than type I IFN as it selectively targets the respiratory tissues reducing the toxic side effects of the treatment observed with type I IFN.

\section{Acknowledgements}

This study is supported by a New Scholar grant from the Ellison Medical Foundation. We thank the Institute of Clinical and Translational Science (ICTS), UCI for providing blood from young donors.

\section{References}

1 Elliot AJ, Fleming DM: Influenza and respira- 12 Panda A, Qian F, Mohanty S, van Duin D, tory syncytial virus in the elderly. Expert Rev Vaccines 2008;7:249-258.

2 Garcia-Sastre A, Biron CA: Type 1 interferons and the virus-host relationship: a lesson in detente. Science 2006;312:879-882.

3 Gilliet M, Cao W, Liu YJ: Plasmacytoid dendritic cells: sensing nucleic acids in viral infection and autoimmune diseases. Nat Rev Immunol 2008;8:594-606.

4 Honda K, Yanai H, Negishi H, Asagiri M, Sato M, Mizutani T, Shimada N, Ohba Y, Takaoka A, Yoshida N, Taniguchi T: IRF-7 is the master regulator of type-I interferon-dependent immune responses. Nature 2005;434:772777.

5 Shodell M, Siegal FP: Circulating, interferonproducing plasmacytoid dendritic cells decline during human ageing. Scand J Immunol 2002;56:518-521.

6 Perez-Cabezas B, Naranjo-Gomez M, Fernandez MA, Grifols JR, Pujol-Borrell R, Borras FE: Reduced numbers of plasmacytoid dendritic cells in aged blood donors. Exp Gerontol 2007;42:1033-1038.

7 Jing Y, Shaheen E, Drake RR, Chen N, Gravenstein S, Deng Y: Aging is associated with a numerical and functional decline in plasmacytoid dendritic cells, whereas myeloid dendritic cells are relatively unaltered in human peripheral blood. Hum Immunol 2009;70: 777-784.

8 Canaday DH, Amponsah NA, Jones L, Tisch DJ, Hornick TR, Ramachandra L: Influenzainduced production of interferon-alpha is defective in geriatric individuals. J Clin Immunol 2010;30:373-383.

9 Agrawal A, Agrawal S, Cao JN, Su H, Osann K, Gupta S: Altered innate immune functioning of dendritic cells in elderly humans: a role of phosphoinositide 3-kinase-signaling pathway. J Immunol 2007;178:6912-6922.

10 Sridharan A, Esposo M, Kaushal K, Tay J, Osann K, Agrawal S, Gupta S, Agrawal A: Age-associated impaired plasmacytoid dendritic cell functions lead to decreased CD4 and CD8 T cell immunity. Age (Dordr) 2011; 33:363-376.

11 Della Bella S, Bierti L, Presicce P, Arienti R, Valenti M, Saresella M, Vergani C, Villa ML: Peripheral blood dendritic cells and monocytes are differently regulated in the elderly. Clin Immunol 2007;122:220-228. Newman FK, Zhang L, Chen S, Towle V, Belshe RB, Fikrig E, Allore HG, Montgomery RR, Shaw AC: Age-associated decrease in TLR function in primary human dendritic cells predicts influenza vaccine response. J Immunol 2010;184:2518-2527.

13 Cao W, Manicassamy S, Tang H, Kasturi SP, Pirani A, Murthy N, Pulendran B: Toll-like receptor-mediated induction of type I interferon in plasmacytoid dendritic cells requires the rapamycin-sensitive $\mathrm{PI}(3) \mathrm{K}$-mTORp70s6K pathway. Nat Immunol 2008;9:11571164.

14 Stout-Delgado HW, Yang X, Walker WE, Tesar BM, Goldstein DR: Aging impairs IFN regulatory factor 7 up-regulation in plasmacytoid dendritic cells during TLR9 activation. J Immunol 2008;181:6747-6756.

15 Qian F, Wang X, Zhang L, Lin A, Zhao H, Fikrig E, Montgomery RR: Impaired interferon signaling in dendritic cells from older donors infected in vitro with West Nile virus. J Infect Dis 2011;203:1415-1424.

16 Uno K, Yagi K, Yoshimori M, Tanigawa M, Yoshikawa T, Fujita S: IFN production ability and healthy ageing: mixed model analysis of a 24 year longitudinal study in Japan. BM) Open 2013;3.

17 Agrawal A, Tay J, Ton S, Agrawal S, Gupta S: Increased reactivity of dendritic cells from aged subjects to self-antigen, the human DNA. J Immunol 2009;182:1138-1145.

18 Prakash S, Agrawal S, Cao JN, Gupta S, Agrawal A: Impaired secretion of interferons by dendritic cells from aged subjects to influenza: role of histone modifications. Age (Dordr) 2012, epub ahead of print.

19 Gonzalez-Navajas JM, Lee J, David M, Raz E: Immunomodulatory functions of type I interferons. Nat Rev Immunol 2012;12:125-135.

20 Kolumam GA, Thomas S, Thompson LJ, Sprent J, Murali-Krishna K: Type I interferons act directly on CD8 T cells to allow clonal expansion and memory formation in response to viral infection. J Exp Med 2005;202: 637-650.

21 Heitger A: Regulation of expression and function of IDO in human dendritic cells. Curr Med Chem 2011;18:2222-2233.

22 Hoshi M, Saito K, Hara A, Taguchi A, Ohtaki H, Tanaka R, Fujigaki H, Osawa Y, Takemura $\mathrm{M}$, Matsunami H, Ito H, Seishima M: The ab- sence of IDO upregulates type I IFN production, resulting in suppression of viral replication in the retrovirus-infected mouse. J Immunol 2010;185:3305-3312.

23 Scheler M, Wenzel J, Tuting T, Takikawa O, Bieber T, von Bubnoff D: Indoleamine 2,3-dioxygenase (IDO). The antagonist of type I interferon-driven skin inflammation? Am J Pathol 2007;171:1936-1943.

24 Mozzillo N, Ascierto P: Reduction of circulating regulatory $\mathrm{T}$ cells by intravenous highdose interferon alfa-2b treatment in melanoma patients. Clin Exp Metastasis 2012;29: 801-805.

25 Hoshi M, Matsumoto K, Ito H, Ohtaki H, Arioka Y, Osawa Y, Yamamoto Y, Matsunami H, Hara A, Seishima M, Saito K: L-Tryptophan-kynurenine pathway metabolites regulate type I IFNs of acute viral myocarditis in mice. J Immunol 2012;188:3980-3987.

26 Pertovaara M, Raitala A, Lehtimaki T, Karhunen PJ, Oja SS, Jylha M, Hervonen A, Hurme M: Indoleamine 2,3-dioxygenase activity in nonagenarians is markedly increased and predicts mortality. Mech Age Dev 2006; 127:497-499.

27 Pawelec G, Barnett Y, Forsey R, Frasca D, Globerson A, McLeod J, Caruso C, Franceschi C, Fulop T, Gupta S, Mariani E, Mocchegiani E, Solana R: T cells and aging, January 2002 update. Front Biosci 2002;7:d1056-d1183.

28 Zhou Z, Hamming OJ, Ank N, Paludan SR, Nielsen AL, Hartmann R: Type III interferon (IFN) induces a type I IFN-like response in a restricted subset of cells through signaling pathways involving both the Jak-STAT pathway and the mitogen-activated protein kinases. J Virol 2007;81:7749-7758.

29 Ank N, Iversen MB, Bartholdy C, Staeheli P, Hartmann R, Jensen UB, Dagnaes-Hansen F, Thomsen AR, Chen Z, Haugen H, Klucher K, Paludan SR: An important role for type III interferon (IFN-lambda/IL-28) in TLR-induced antiviral activity. J Immunol 2008;180: 2474-2485.

30 Koltsida O, Hausding M, Stavropoulos A, Koch S, Tzelepis G, Ubel C, Kotenko SV, Sideras P, Lehr HA, Tepe M, Klucher KM, Doyle SE, Neurath MF, Finotto S, Andreakos E: IL-28a (IFN-lambda2) modulates lung DC function to promote TH1 immune skewing and suppress allergic airway disease. EMBO Mol Med 2011;3:348-361. 